\title{
A RELAXED PICARD ITERATION PROCESS FOR SET-VALUED OPERATORS OF THE MONOTONE TYPE
}

\author{
J. C. DUNN
}

\begin{abstract}
The fixed points $\bar{x}$ of set-valued operators, $T: X \rightarrow 2^{X}$, satisfying a condition of the monotonicity type on convex subsets $X$ of a Hilbert space are approximated by a relaxation process, $x_{n+1}=x_{n}+\omega_{n}\left(T x_{n}-x_{n}\right)$, in which $\tilde{T}$ is a single-valued branch of $T$ and the relaxation parameter $\omega_{n} \in[0,1]$ is made to depend in a certain way on the prior history of the process. If $\tilde{T}$ is bounded on bounded subsets of $X$, then $\left\|x_{n}-\bar{x}\right\|$ converges to 0 like $O\left(n^{-1 / 2}\right)$. If $\tilde{T}$ is also continuous at $\bar{x}$ and if $\bar{x}=\bar{T} \bar{x}$, then $\left\|x_{n}-\bar{x}\right\|=o\left(n^{-1 / 2}\right)$. If $\tilde{T}$ satisfies a condition of the Lipschitz type at $\bar{x}$, then $\left\|x_{n}-\bar{x}\right\|=O\left(\mu^{n / 2}\right)$ for some $\mu \in[0,1)$.
\end{abstract}

1. Introduction. Let $X$ be a nonempty convex subset of a Hilbert space $H$, and suppose that for some $\bar{x} \in X$, the set-valued map $T: X \rightarrow 2^{x}$ satisfies the monotonicity condition,

$$
1>C_{*} \triangleq \sup _{\substack{x \in X \\ x \neq \bar{x}}} \sup _{\xi \in T x} \frac{\operatorname{Re}\langle\xi-\bar{x}, x-\bar{x}\rangle}{\|x-\bar{x}\|^{2}} ;
$$

(every fixed point of $T$ necessarily coincides with $\bar{x}$ ). Suppose also that the range of $T$ is bounded in the sense that

$$
\operatorname{diam} \bigcup_{x \in X} T x<\infty
$$

and therefore,

$$
\sup _{x \in X} \sup _{\xi \in T x}\|\xi-\bar{x}\|<\infty .
$$

If $\tilde{T}$ is any single-valued branch of $T$ and if $\left\{x_{n}\right\} \subset X$ is generated by the associated relaxation process,

$$
x_{n+1}=x_{n}+\omega_{n}\left(\tilde{T} x_{n}-x_{n}\right), \quad x_{0} \in X,
$$

where the relaxation parameter sequence $\left\{\omega_{n}\right\} \subset[0,1]$ satisfies

$$
\sum_{n=0}^{\infty} \omega_{n}^{2}<\infty
$$

Received by the editors December 27, 1977 and, in revised form, June 1, 1978.

AMS (MOS) subject classifications (1970). Primary 47H05, 47H10; Secondary 65J05, 68A10.

Key words and phrases. Set-valued Hilbert space operators, fixed points, relaxed Picard iterations, residual-dependent step lengths, continuity conditions and convergence rates.

(C) 1979 American Mathematical Society 0002-9939/79/0000-0106/\$03.25 
and

$$
\sum_{n=0}^{\infty} \omega_{n}=\infty .
$$

Then $\left\{x_{n}\right\}$ converges strongly to $\bar{x}$. Moreover, for certain special parameter sequences of this type, one can show that $\left\|x_{n}-\bar{x}\right\|=O\left(n^{-1 / 2}\right)[1],[2],[3]$ (for related results see [4] also). To construct such a sequence, it is enough to have a value of $C$ satisfying $1>C \geqslant C_{*}$; no additional information about $T$ or the course of the iteration (1.4) is required, hence the parameter sequence $\left\{\omega_{n}\right\}$ can be computed before (1.4) commences.

Roughly speaking, condition (1.5) requires that $\left\{\omega_{n}\right\}$ converge to 0 but not "too quickly". This restriction is necessary if (1.4) is to work in the worst cases where $\tilde{T}$ is discontinuous at $\bar{x}$. Without continuity, it is possible for the residual norms $\left\|\tilde{T} x_{n}-x_{n}\right\|$ to remain bounded away from zero while $x_{n}$ converges to a fixed point $\bar{x}$ of $\tilde{T}$, and this behavior is generally incompatible with (1.4) unless $\omega_{n} \rightarrow 0$. Unfortunately, while (1.5) insures convergence in the worst cases, it virtually guarantees a uniformly poor asymptotic rate of convergence in all cases, even where $\tilde{T}$ is Lipschitz continuous. On the other hand, it is known that for Lipschitz continuous $\tilde{T}$ satisfying (1.1), the sequences $\left\{x_{n}\right\} \subset X$ generated by (1.4) will converge geometrically, or linearly, to $\bar{x}$ (i.e., $\left\|x_{n}-\bar{x}\right\|=O\left(\lambda^{n}\right.$ ) for some $\lambda \in[0,1)$ ), provided the terms in $\left\{\omega_{n}\right\}$ are sufficiently small but at the same time bounded away from $0[5]-[9]$. In fact, let $\tilde{T}$ satisfy the condition

$$
K_{*} \triangleq \sup _{\substack{x \in X \\ x \neq \bar{x}}} \frac{\|\tilde{T} x-\bar{x}\|}{\|x-\bar{x}\|}<\infty .
$$

For $\omega_{n} \in[0,1],(1.1)$ and (1.4) then give

$$
\left\|x_{n+1}-\bar{x}\right\|^{2} \leqslant \lambda_{*}\left(\omega_{n}\right)\left\|x_{n}-\bar{x}\right\|^{2} \leqslant \lambda\left(\omega_{n}\right)\left\|x_{n}-\bar{x}\right\|^{2}
$$

with

$$
\lambda_{*}(\omega)=1-2\left(1-C_{*}\right) \omega+\left(1-2 C_{*}+K_{*}^{2}\right) \omega^{2}
$$

and

$$
\lambda(\omega)=1-2(1-C) \omega+\left(1-2 C+K^{2}\right) \omega^{2}
$$

where

$$
1>C \geqslant C_{*}
$$

and

$$
K \geqslant K_{*} \text {. }
$$

If for all $n>0, \omega_{n}=\omega$ with $\omega$ positive and sufficiently small, the parameter $\lambda\left(\omega_{n}\right)$ (and a fortiori $\lambda_{*}\left(\omega_{n}\right)$ ) will be nonnegative and smaller than 1 , hence $\left\{x_{n}\right\}$ will converge to $\bar{x}$ geometrically. Furthermore for any given bounds 
$C \geqslant C_{*}$ and $K \geqslant K_{*}$, there is a "best" value $\omega=\omega_{\mathrm{OPT}}$ which minimizes the parameter $\lambda(\omega)$ in the bound (1.7A). To see this, observe first that for fixed $K$ and fixed $\omega \in[0,1], \lambda(\omega)$ increases with increasing $C$. Furthermore, since the Schwarz inequality, (1.1) and (1.6) give $\left|C_{*}\right| \leqslant K_{*}$, it follows that $K \geqslant K_{*}$ is always a better estimate of $C_{*}$ than any $C>K$. Therefore, consideration may be limited to $C$ 's in the range,

$$
|C| \leqslant K \text { and } C<1 .
$$

In this event, the coefficient of $\omega^{2}$ in $(1.7 C)$ is always positive and $\lambda(\omega)$ attains its least value over $\omega \in[0,1]$ at

$$
\omega_{\mathrm{OPT}}=\min \left\{1, \frac{1-C}{1-2 C+K^{2}}\right\}
$$

where

$$
\lambda_{\mathrm{OPT}}=\lambda\left(\omega_{\mathrm{OPT}}\right)=\left\{\begin{array}{l}
K^{2}, \quad \text { if }(1-C) \geqslant 1-2 C+K^{2}, \\
\frac{\left(K^{2}-C^{2}\right)}{(1-C)^{2}+\left(K^{2}-C^{2}\right)}, \quad \text { if }(1-C)<1-2 C+K^{2} .
\end{array}\right.
$$

In either case,

$$
0 \leqslant \lambda_{* \mathrm{OPT}} \leqslant \lambda_{\mathrm{OPT}}<1
$$

where $\omega_{* \mathrm{OPT}}$ and $\lambda_{* \mathrm{OPT}}$ are obtained by putting $C=C^{*}$ and $K=K_{*}$ in $(1.8 \mathrm{~A})$ and $(1.8 \mathrm{~B})$. These estimates show that asymptotic convergence rates far better than $O\left(n^{-1 / 2}\right)$ are attainable with (1.4) in principle when $\tilde{T}$ is sufficiently continuous at $\bar{x}$. However, in order to apply the relaxation parameter rule (1.8), one must have values for $C$ and $K$ which satisfy (1.7D) and (1.7E); if (1.8) is used with a $K<K_{*}$ or $C \notin\left[C_{*}, 1\right)$, then $\tilde{T}_{\omega_{\text {OPT }}}=I+$ $\omega_{\text {OPT }}(\tilde{T}-I)$ is not necessarily contractive and the corresponding sequence $\left\{x_{n}\right\}$ generated by (1.4) with $\omega_{n}=\omega_{\text {OPT }}$ may not converge to any limit.

The present article considers a family of iterative processes (1.4) in which $\omega_{n}$ is made to depend in a simply way on the prior history of $x_{k}$, for $0 \leqslant k \leqslant n$. If $T$ is set-valued and satisfies the monotonicity condition (1.1), the corresponding error norms $\left\|x_{n}-\bar{x}\right\|$ are of order $O\left(n^{-1 / 2}\right)$ as $n \rightarrow \infty$ for any branch $\tilde{T}$ that is bounded on bounded subsets of $X . T$ need not satisfy the stronger boundedness condition (1.2) invoked in [1] and need not be continuous for this estimate to apply, however if $\tilde{T}$ is continuous at $\bar{x}$ and if $\bar{x}=\tilde{T} \bar{x}$, it turns out that $\left\|x_{n}-\bar{x}\right\|=o\left(n^{-1 / 2}\right)$. Moreover, if $\tilde{T}$ satisfies condition (1.6) at $\bar{x}$, then the error norms converge to 0 geometrically, even though neither the constant $K_{*}$ nor an upper bound $K$ for $K_{*}$ actually appears in the rule which determines $\omega_{n}$ (and is therefore not required for an implementation of this rule).

In [10], Williamson considers a relaxation process (1.4) for $T: X \rightarrow 2^{H}$, with 
$\omega_{n}$ depending on $x_{n}$ according to a rule which turns out to be essentially a special case of the scheme proposed here; strong convergence of $x_{n}$ to $\bar{x}$ is established when $X$ is dense in $H$ (or in a solid sphere $\subset H$ ), and when $T$ is bounded on bounded subsets of $X$. Useful a posteriori error estimates are also derived in [10], along with the basic $O\left(n^{-1 / 2}\right)$ asymptotic convergence rate estimate.

The principal analytical tools employed in the next section were developed in [11] for a different purpose.

\section{Results. Put}

$$
R_{n}=\tilde{T} x_{n}-x_{n} \text { and } E_{n}=x_{n}-\bar{x} .
$$

Then (1.4) yields

$$
E_{n+1}=E_{n}+\omega_{n} R_{n}
$$

and it follows easily from the monotonicity condition (1.1) that for all $n \geqslant 0$ and $\omega_{n}>0$,

$$
\frac{\left\|E_{n+1}\right\|^{2}}{\theta^{2}} \leqslant\left[1-2(1-C) \omega_{n}\right] \frac{\left\|E_{n}\right\|^{2}}{\theta^{2}}+\frac{\omega_{n}^{2}}{\theta^{2}}\left\|R_{n}\right\|^{2}
$$

where $\theta^{2} \neq 0$ is a scaling parameter about which more will be said later, and where $1>C>C_{*}$.

Let

$$
\bar{\omega}=\min \left\{1, \frac{1}{2(1-C)}\right\}>0
$$

and suppose that

$$
0 \leqslant \omega_{n} \leqslant \bar{\omega} \leqslant 1
$$

for all $n>0$. Then the iterates $\left\{x_{n}\right\}$ generated by (1.4) cannot leave the convex set $X$. Moreover, the bracketed term on the right side of (2.1) is never negative and consequently a simple induction yields

$$
0 \leqslant\left\|E_{n}\right\|^{2} \leqslant B \beta_{n}
$$

for all $n>0$, where,

$$
B=\max \left\{\theta^{2},\left\|E_{0}\right\|\right\}
$$

and the sequence $\left\{\beta_{n}\right\} \subset[0, \infty)$ is recursively generated by

$$
\beta_{n+1}=\left[1-2(1-C) \omega_{n}\right] \beta_{n}+\frac{\omega_{n}^{2}}{\theta^{2}}\left\|R_{n}\right\|^{2}, \quad \beta_{0}=1 .
$$

Since the error estimates (2.3) hold for any step length sequence $\left\{\omega_{n}\right\}$ 
satisfying (2.2), they are valid when $\omega_{n}$ is determined by the rule,

$$
\omega_{n}=\tilde{\omega}\left(x_{n}, \beta_{n} ; \theta\right)=\left\{\begin{array}{l}
0, \quad \text { if } R_{n}=0, \\
\min \left\{\bar{\omega}, \frac{\theta^{2}(1-C) \beta_{n}}{\left\|R_{n}\right\|^{2}}\right\}, \quad \text { if } R_{n} \neq 0 .
\end{array}\right.
$$

This scheme insures that $\left\{x_{n}\right\}$ will terminate at $x_{N}$ if $x_{N}$ is a fixed point of $\tilde{T}$, and otherwise minimizes the right side of $(2.3 \mathrm{C})$ over $\omega_{n} \in[0, \bar{\omega}]$, given $\beta_{n}$ and $R_{n}$.

LEMMA 2.1. If the sequences $\left\{\omega_{n}\right\},\left\{\beta_{n}\right\}$, and $\left\{\left\|R_{n}\right\|\right\}$ satisfy (2.3C) and (2.4) for $n \geqslant 0$, then $\left\{\beta_{n}\right\}$ is nonnegative and monotone nonincreasing.

Proof. It has already been shown that $\beta_{n}>0$ for all $n \geqslant 0$. When $\omega_{n}=0$ in (2.4), (2.3C) $\Rightarrow \beta_{n+1}=\beta_{n}$; when $\omega_{n}=\theta^{2}(1-C) \beta_{n} /\left\|R_{n}\right\|^{2}$,

$$
(2.3 \mathrm{C}) \Rightarrow \beta_{n+1}=\left(1-\frac{\theta^{2}(1-C)^{2} \beta_{n}}{\left\|R_{n}\right\|^{2}}\right) \beta_{n} \leqslant \beta_{n} ;
$$

finally, when $\omega_{n}=\bar{\omega}$,

$$
\begin{aligned}
(2.3 C) & \Rightarrow \beta_{n+1}=[1-2(1-C) \bar{\omega}] \beta_{n}+\bar{\omega}^{2} \frac{\left\|R_{n}\right\|^{2}}{\theta^{2}} \\
& \leqslant[1-(1-C) \bar{\omega}] \beta_{n} \leqslant \max \left\{C, \frac{1}{2}\right\} \beta_{n}<\beta_{n} .
\end{aligned}
$$

In all cases, $\beta_{n+1} \leqslant \beta_{n}$. Q.E.D.

Corollary 1. Let $T: X \rightarrow 2^{X}$ satisfy the monotonicity condition (1.1), let the single-valued branch $\tilde{T}$ in (1.4) be bounded on bounded subsets of $X$, and let $\left\{x_{n}\right\} \subset X$ be generated by (1.4)-(2.4). Then $\left\{x_{n}\right\}$ and the associated residual sequence $\left\{R_{n}\right\}$ are bounded.

Proof. $\left\{\beta_{n}\right\}$ is monotone nonincreasing and (2.3A) and (2.3B) hold, therefore $\left\|x_{n}-\bar{x}\right\|<B^{1 / 2}$ for all $n \geqslant 0$. Since $T$ is bounded on bounded subsets, the sequence $\left\{\tilde{T} x_{n}-\bar{x}\right\}$ is also bounded. Boundedness of $\left\{T x_{n}-\right.$ $\left.x_{n}\right\}$ is now immediate from the triangle inequality. Q.E.D.

LEMMA 2.2. Let $\left\{\beta_{n}\right\} \subset(0, \infty)$ and $\left\{q_{n}\right\} \subset[0, \infty)$ satisfy

$$
\beta_{n+1} \leqslant \beta_{n}-q_{n} \beta_{n}^{2} ; \quad \beta_{0}=1
$$

for all $n>0$. If $q_{n} \geqslant q>0$ for $n \geqslant 0$, then

$$
0<\beta_{n} \leqslant \frac{1}{1+q n}=O\left(n^{-1}\right) .
$$

Furthermore, if $\lim _{n \rightarrow \infty} q_{n}=\infty$, then $\beta_{n}=o\left(n^{-1}\right)$. 
Proof. ${ }^{1}$ Put $\delta_{k}=1 / \beta_{k}$. Then for all $k \geqslant 0$, one has $\delta_{k}-q_{k}>0$ and $\delta_{k+1}-\delta_{k} \geqslant q_{k} \delta_{k} /\left(\delta_{k}-q_{k}\right) \geqslant q_{k}$. Consequently, for all $n \geqslant 0$,

$$
\delta_{n}=\delta_{0}+\sum_{k=0}^{n-1}\left(\delta_{k+1}-\delta_{k}\right) \geqslant 1+\sum_{k=0}^{n-1} q_{k} \text {. }
$$

If $q_{k} \geqslant q$ for all $k$, one then obtains (2.6). If $q_{n} \rightarrow \infty$, then for any $M>0$ there is an $L$ so large that $k \geqslant L \Rightarrow q_{k} \geqslant M$. For $n>L$ one then has

$$
\begin{aligned}
0 & <n \beta_{n}=\frac{n}{\delta_{n}} \\
& \leqslant \frac{n}{1+\sum_{k=0}^{L-1} q_{k}+\sum_{k=L}^{n-1} q_{k}} \leqslant \frac{n}{(n-L) M}
\end{aligned}
$$

and therefore $0 \leqslant \underline{\lim }_{n \rightarrow \infty} n \beta_{n} \leqslant \varlimsup_{n} n \beta_{n} \leqslant 1 / M$. Since $M$ can be arbitrarily large, it follows that $\lim _{n \rightarrow \infty} n \beta_{n}=0$. Q.E.D.

THEOREM 2.1. Let $X$ be a nonempty convex subset of a Hilbert space $H$, let $T: X \rightarrow 2^{X}$ satisfy (1.1), and let $\tilde{T}$ be a single-valued branch of $T$ that is bounded on bounded subsets of $X$. Suppose that $\left\{x_{n}\right\} \subset X,\left\{\beta_{n}\right\} \subset[0, \infty)$, and $\left\{\omega_{n}\right\} \subset[0, \bar{\omega}]$ are generated by (1.4), (2.3C), and (2.4) with any $\theta \neq 0, C \in$ $\left[C^{*}, 1\right)$, and $\bar{\omega}=\min \{1,1 / 2(1-C)\}$. Let $\left\{R_{n}\right\}$ and $\left\{E_{n}\right\}$ denote the associated residual sequence and error sequence in (2.1). Then $\left\{R_{n}\right\}$ is bounded and for all $n \geqslant 0$,

$$
0 \leqslant\left\|E_{n}\right\|^{2} \leqslant \max \left\{\theta^{2},\left\|E_{0}\right\|^{2}\right\} \cdot \beta_{n}
$$

with $\left\{\beta_{n}\right\}$ nonnegative and monotone nonincreasing. Furthermore, either there is an $N \geqslant 0$ such that

$$
x_{n}=\bar{x}, \quad \forall n \geqslant N
$$

or else

$$
0<\beta_{n} \leqslant \frac{1}{1+q n}, \quad \forall n \geqslant 0
$$

with

$$
\begin{gathered}
q=\min \left\{A, \frac{\theta^{2}(1-C)^{2}}{d^{2}}\right\} \\
A=1-\max \left\{\frac{1}{2}, C\right\}
\end{gathered}
$$

and

$$
\infty>d \geqslant d_{*} \triangleq \sup _{n>0}\left\|R_{n}\right\|
$$

in which case $\left\|E_{n}\right\|=O\left(n^{-1 / 2}\right)$. Finally, if $\bar{x}$ is a fixed point of $\tilde{T}$ and if $\tilde{T}$ is continuous at $\bar{x}$, then $\left\|x_{n}-\bar{x}\right\|=o\left(n^{-1 / 2}\right)$.

'This result is established in [7]; for convenience, the proof is repeated here. 
Proof. Corollary 1 of Lemma $2.1 \Rightarrow\left\{R_{n}\right\}$ is bounded. (2.7) has already been established in (2.3). Lemma $2.1 \Rightarrow\left\{\beta_{n}\right\}$ nonnegative and monotone nonincreasing. If $R_{n}=0$, then $x_{n}$ is a fixed point of $\tilde{T}$ and must coincide with $\bar{x}$ because of (1.1) [1]. Furthermore, (1.4)-(2.4) $\Rightarrow \omega_{n}=0$ and $x_{n+1}=x_{n}=\bar{x}$. (2.8) now follows by induction.

If $R_{n} \neq 0$ and $\beta_{n}>0$, then (2.3C) and (2.4) give

$$
0<\beta_{n+1} \leqslant\left(1-q_{n} \beta_{n}\right) \beta_{n}
$$

with

$$
q_{n}=\min \left\{\frac{A}{\beta_{n}}, \frac{\theta^{2}(1-C)^{2}}{\left\|R_{n}\right\|^{2}}\right\} \geqslant q .
$$

Since $\beta=1$, one obtains $\beta_{n}>0$ for all $n \geqslant 0$ by induction if $R_{n} \neq 0$ for all $n \geqslant 0$. The estimate (2.9) is now immediate from Lemma 2.2.

Finally if $\bar{x}=\tilde{T} \bar{x}$, if $\tilde{T}$ is continuous at $\bar{x}$, and if $R_{n} \neq 0$ for all $n \geqslant 0$, then (2.7) and (2.9) $\Rightarrow\left\|R_{n}\right\| \rightarrow 0$ and $\beta_{n} \rightarrow 0$ through positive values, in which case (2.10B) $\Rightarrow \lim _{n \rightarrow \infty} q_{n}=\infty$. It now follows from (2.7), (2.10A) and Lemma 2.2 that $\left\|E_{n}\right\|=o\left(n^{-1 / 2}\right)$. Q.E.D.

THEOREM 2.2. Let the hypothesis of Theorem 2.1 hold and suppose in addition that $\tilde{T}$ satisfies (1.6). If $R_{n} \neq 0$ for $0 \leqslant n<N$, and $R_{N}=0$, then (2.8) holds, and for $0 \leqslant n \leqslant N$,

$$
0<\beta_{n} \leqslant \mu^{n}
$$

with

$$
\begin{aligned}
\mu & =1-\min \left\{A, \frac{\theta^{2}(1-C)}{B\left(K_{*}+1\right)^{2}}\right\} \leqslant 1-\min \left\{A, \frac{\theta^{2}(1-C)}{B(K+1)^{2}}\right\}<1, \\
A & =1-\max \left\{\frac{1}{2}, C\right\} \\
B & =\max \left\{\theta^{2},\left\|E_{0}\right\|^{2}\right\}
\end{aligned}
$$

and

$$
K \geqslant K_{*}
$$

If $R_{n} \neq 0$ for all $n \geqslant 0$, then (2.11) holds for all $n \geqslant 0$ and it follows from (2.7) that $\left\|E_{n}\right\|=O\left(\mu^{n / 2}\right)$.

Proof. If $R_{n} \neq 0$ for $0 \leqslant n<N$, then $\beta_{n}$ is positive and satisfies (2.10) for $0 \leqslant n \leqslant N$. Furthermore,

$$
\begin{aligned}
(1.6) & \Rightarrow\left\|R_{n}\right\|^{2}=\left\|T x_{n}-x_{n}\right\|^{2} \\
& \leqslant\left(\left\|T x_{n}-\bar{x}\right\|+\left\|x_{n}-\bar{x}\right\|\right)^{2} \leqslant(K+1)^{2}\left\|E_{n}\right\|^{2}
\end{aligned}
$$


and therefore (2.7) and (2.10) give

$$
\frac{\theta^{2}(1-C)^{2} \beta_{n}}{\left\|R_{n}\right\|^{2}} \geqslant \frac{\theta^{2}(1-C)^{2}}{B(K+1)^{2}}
$$

and

$$
0<1-q_{n} \beta_{n} \leqslant \mu
$$

for $0 \leqslant n \leqslant N$. Q.E.D.

Note 2.1. Theorems 2.1 and 2.2 hold for any nonzero value of the scaling parameter $\theta$. If $E$ is any upper bound on $\left\|E_{0}\right\|$, then (2.7) and (2.8) give the estimate

$$
\left\|E_{n}\right\|^{2} \leqslant \kappa(\theta) \cdot n^{-1}
$$

with

$$
\kappa(\theta)=\max \left\{\theta^{2}, E^{2}\right\} \max \left\{\frac{1}{A}, \frac{d^{2}}{\theta^{2}(1-C)^{2}}\right\} .
$$

The coefficient $\kappa(\theta)$ always attains its least value when $\theta^{2}=E^{2}$; for this value of $\theta$, one has

$$
\left\|E_{n}\right\|^{2} \leqslant \max \left\{\frac{E^{2}}{A}, \frac{d^{2}}{(1-C)^{2}}\right\} \cdot n^{-1} .
$$

Similarly, if $E$ bounds $\left\|E_{0}\right\|$, then (2.7) and (2.11) yield

$$
\left\|E_{n}\right\|^{2} \leqslant \max \left\{\theta^{2}, E^{2}\right\}(\bar{\mu}(\theta))^{n}
$$

with

$$
\bar{\mu}(\theta)=1-\min \left\{A, \frac{(1-C)}{\left(K_{*}+1\right)^{2} \max \left\{E^{2} / \theta^{2}, 1\right\}}\right\} .
$$

Once again, $\theta^{2}=E^{2}$ yields the sharpest of these bounds, namely

$$
\left\|E_{n}\right\|^{2} \leqslant E^{2}(\bar{\mu})^{n}
$$

with

$$
\bar{\mu}=1-\min \left\{A,(1-C) /\left(K_{*}+1\right)^{2}\right\} .
$$

Note 2.2. If $X=H$ (or a linear variety in $H$ ) and if $T: X \rightarrow 2^{X}$, there is no reason for restricting $\omega_{n}$ to the interval $[0,1]$, and Theorems 2.1 and 2.2 remain valid if $\bar{\omega}$ is replaced by $1 / 2(1-C)$ in (2.4) and $A$ is replaced by $\frac{1}{2}$ in (2.9).

Note 2.3. Williamson has shown in [10] that

$$
\left\|E_{0}\right\|<E \stackrel{\Delta}{=}\|T y-y\| / 2(1-C)
$$


if

$$
x_{0}=y+(T y-y) / 2(1-C)
$$

for some $y \in X$. The estimate (2.12) can also be obtained by putting $\omega=$ $1 / 2(1-C)$ in the inequality

$$
\left\|x_{0}-\bar{x}\right\|^{2} \leqslant[1-2(1-C) \omega]\|y-\bar{x}\|^{2}+\omega^{2}\|T y-y\|^{2}, \quad \forall \omega \geqslant 0
$$

which is gotten by the same argument that produces $(2.1)$. For the case $X=H$, put $\bar{\omega}=1 / 2(1-C)$ in the rule (2.4) (see Note 2.2). Furthermore, let $x_{0}$ satisfy (2.12B) and set $\theta^{2}=E^{2}$, with $E$ given by (2.12A). Then (1.4)-(2.4) reduces to the basic iteration scheme in [10], with error bounds $r_{i}$ related to the $\beta_{i}$ 's in (2.3A) by

$$
r_{i+1}^{2}=E^{2} \beta_{i}, \quad \forall i \geqslant 0
$$

(see Note 2.1).

\section{REFERENCES}

1. J. C. Dunn, Iterative construction of fixed points for multivalued operators of the monotone type, J. Functional Analysis 27 (1978), 38-50.

2. R. E. Bruck, Jr., The iterative solution of the equation $y \in x-T x$ for a monotone operator $T$ in Hilbert space, Bull. Amer. Math. Soc. 79 (1973), 1258-1261.

3. B. E. Rhoades, Fixed point iterations using infinite matrices. III, in Proc. Conf. on Computing Fixed Points with Applications (Clemson Univ., June 1974), Academic Press, New York (to appear).

4. R. E. Bruck, A strongly convergent iterative solution of $0 \in U(x)$ for a maximal monotone operator $U$ in Hilbert space, J. Math. Anal. Appl. 48 (1974), 114-126.

5. E. H. Zarantonello, Solving functional equations by contractive averaging, U. S. Army Mathematics Research Center Technical Report $\# 160$, Wisconsin, June 1960.

6. $\ldots$ The closure of the numerical range contains the spectrum, Bull. Amer. Math. Soc. 70 (1964), 781-787.

7. The closure of the numerical range contains the spectrum, Pacific J. Math. 22 (3) (1967), 575-595.

8. A. B. Bakusinskii and B. T. Poljak, On the solution of variational inequalities, Soviet Math. Dokl. 15 (6) (1974), 1705-1710.

9. F. E. Browder and W. V. Petryshyn, Construction of fixed points of nonlinear mappings in Hilbert space, J. Math. Anal. Appl. 20 (1967), 197-228.

10. T. E. Williamson, Jr., Geometric estimation of $x=T x=0$ for monotone-type operator $T$ in Hilbert space, Proc. Amer. Math. Soc. (to appear).

11. J. C. Dunn, Rates of convergence for conditional gradient algorithms near singular and nonsingular extremals, SIAM J. Control Optimization (to appear).

\footnotetext{
Department of Mathematics, North Carolina State University, Raleigh, North Carolina 27650
} 